\title{
THE LIGHT CURVE OF V444 CYGNI
}

\author{
W.-R. HAMANN, E. SCHWARZ, U. WESSOLOWSKI \\ Institut für Theoretische Physik und Sternwarte der Universität, \\ Olshausenstraße 40, D-2300 Kiel, Federal Republic of Germany
}

In order to synthesize the eclipse light curve of V444 Cygni, we adopt the following model. The $O$ star revolves round the WR star still within the outer regions of its extended atmosphere. The $O$ star shadows a distinct volume of the WR atmosphere which thus cannot contribute to the total flux seen by the observer. On the other hand, additional radiation emerges from the surface of the $\mathrm{O}$ star. Its contribution to the total flux is more or less diminished by absorption when the rays pass through those parts of the WR atmosphere which lie between the $O$ star and the observer. The WR atmosphere is given by our usual models (cf. Hamann and Schmutz, 1987; Wessolowski et al., 1988).

A couple of free parameters describing the WR star $\left(R_{*}, T_{*}, \dot{M}\right)$, the $O$ star $\left(T_{\text {eff }}, R\right)$, and the orbit (namely, the inclination angle $i$ ) can be varied until the synthetic light curve matches the observation. The mass-loss rate has a very characteristic influence, as it determines the width of the first minimum when the $O$ star is occulted by the extended WR atmosphere. We find $\dot{\mathrm{M}}=10^{-4.9} \mathrm{M}_{\odot} / \mathrm{yr}$.

Apart from $\dot{M}$ there remain five free parameters in order to fit essentially two properties of the light curve, namely the depths of the two minima. It is not likely that there is a unique solution for that problem. In order to find at least one particular solution, we arbitrarily adopt $T_{\text {eff }}=30 \mathrm{kK}(\mathrm{O}$ star), $T$. $=40 \mathrm{kK}$ (WR star) and $i=78^{\circ}$. Then we vary both stellar radii and achieve a good fit of the observed light curve at all wavelengths (Fig. 1). But we can fit the light curve with different sets of parameters as well. E.g., an increase of the inclination $i$ and a corresponding decrease of both radii leads to the same good agreement with observation. For $\mathrm{T}_{*}=60 \mathrm{kK}$ (WR star) we also find a light-curve solution. Thus the basic result is that we can reproduce the observed light curve of V444 Cygni with our model, but the solution is not unique.

Recall that Cherepashchuk et al. (1984) performed a light curve analysis of V444 Cygni and obtained a solution with a "hot" WR star ( $90 \mathrm{kK}$ effective temperature, related to the radius where $\tau=1)$. We suspect that this is another particular solution.

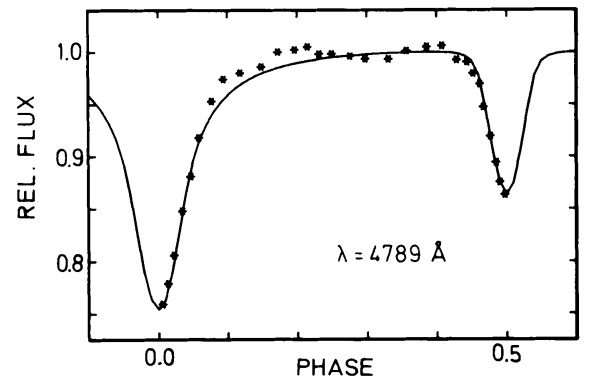

Fig. 1. Light curve of V444 Cygni at $4789 \AA$. Discrete symbols denote observational data, averaged over several periods, as taken from Cherepashchuk and Khalliullin (1973). The synthetic light curve (continuous line) results from a WR model with $\mathrm{R}$. $=6.5 \mathrm{R}_{\odot}, \mathrm{T}_{*}=40 \mathrm{kK}, \dot{\mathrm{M}}=10^{-4.9} \mathrm{M}_{\odot} / \mathrm{yr}$, an O star with $\mathrm{T}_{\text {eff }}=30 \mathrm{kK}, \mathrm{R}=6.7 \mathrm{R}_{\odot}$, and a circular orbit with $a \sin i=40 \mathrm{R}_{\odot}$, and inclination $i=78^{\circ}$. Similar fits are obtained for other spectral bands.

\section{References}

Cherepashchuk, A.M., Khalliullin, K.F.: 1973, Soviet Astr. - AJ 17, 330

Cherepashchuk, A.M., Eaton, J.A., Khalliullin, K.F.: 1984, Ap. J. 281, 774

Hamann, W.-R., Schmutz, W.: 1987, Astr. Ap. 174, 173

Wessolowski, U., Schmutz, W., Hamann, W.-R: 1988, Astr. Ap. 194, 160 